\title{
Research on A Certain Type of Rocket Virtual Training System Based on Unity 3d
}

\author{
Chao Li, Wen-Su Ji, Hong-An Cao, Zhi-Zhi Yan \\ Wuhan N.C.O. Academy of P.L.A., Wuhan, Hubei 430075, China \\ E-mail: lichao062062@21cn.com, wensuji324@163.com, hongan123@sina.com, emilyzhizhi@163.com
}

\begin{abstract}
For the problems that the current virtual reality engine platform versatility is not strong, and the virtual training system is too complex, virtual operation training system is set up based on the concept of $3 D$ games in the article. The key technology of system implementation was studied, including the use of $\mathrm{C}^{\#}$ development general system structure and the Finger Gestures plug-ins implement multitasking gestures interactions. The system is operated smoothly through object processing equipment model and scenario driven programming. The results show that the system can satisfy the requirements of virtual training, which provides a certain reference for realizing virtual operating training on a portable platform.
\end{abstract}

Keywords-computer application; virtual training; unity 3D; common system architecture; gesture touch

\section{INTRODUCTION}

A certain type of missile is a new column of troops guided munition, complex, expensive, and not conducive to conducting live-fire, real soldiers, field operation training, which greatly restricted the generation of the support capability. Therefore we need to carry out the construction of supporting resource conditions.

Unity 3D, in recent years the rapid rise of a professional game engine, has a very big advantage in cross-platform development, and supports almost all major platforms (PC, Web, mobile terminals). And only once developed, it will be able to minimum the cost to deploy to multiple platforms ${ }^{[1-4]}$. If it could be applied to the development of virtual training system, it can certainly be greatly enriched the training route. Now virtual operating interactive aspects in equipment, mostly using the mouse and keyboard or expensive immersive VR peripheral devices (such as HMD, data glove, handles, etc.). Operating virtual objects with the mouse and keyboard way, poor experience, immersive, to some extent, affects the equipment operation training. Using expensive VR peripherals brings new cost problem. Gesture touch technology in recent years, rapid development in the field of human-computer interaction, is very convenient to use. If applied to the virtual operation training, it must be highly beneficial to improve training immersive and enhance the fun ${ }^{[5-7]}$.

Based on this, the author developed a Rocket virtual training system in a portable android platform with the help of Unity 3D engine technology and gesture touch technology, which provides a certain reference for the realization of virtual training on a portable platform.

\section{OVERALL DESIGN OF THE SYSTEM}

Considering that the system eventually run on the android platform, in order to assure that both software runs smoothly, and do not break the pertinence of training, the system uses 3D game design concept, which simplifies the system, keep the main training function, and enhance the interest at the same time. The system mainly consists of the principles of cognitive mode function self-test mode, the theoretical evaluation mode, operating mode of assessment and other functions.

\section{THE KEY TECHNOLOGY OF SYSTEM IMPLEMENTATION}

\section{A. The Implementation of The General System Structure}

Due to the complexity of the type of equipment covered by the training course, the commonality of the structure of the system is very important. Therefore the system adopts the design ideas of "three layer architecture", namely the data layer, logic layer, and separate the UI layer. Define the key such as shown in Table 1. 
TABLE I. TABLE KEY CLASS AND ITS FUNCTIONS

\begin{tabular}{|c|c|c|}
\hline & Key class & Functions \\
\hline 1 & GlobalManager.cs & $\begin{array}{l}\text { Global management class. Identify the current mode, the current state of the system, the } \\
\text { current UI interface display, any class can interact with this class. }\end{array}$ \\
\hline 2 & UIManager.cs & UI management class and UI message processing class. \\
\hline 3 & StepManager.cs & $\begin{array}{l}\text { Step control class. After the flash between two frames is completed, the next thing to do will } \\
\text { expand in this class. }\end{array}$ \\
\hline 4 & StepPlayer.cs & Step class. Play the animation between two frames. \\
\hline 5 & StepUnit.cs & Step data structure. By reading the configuration file generated data structure that is needed. \\
\hline 6 & AnimationPlayer.cs & $\begin{array}{l}\text { Animation player. Play object animations. A variety of load, can provide the functions of } \\
\text { play, pause, stop and play back callback, pause, stop and callback. }\end{array}$ \\
\hline 7 & AudioPlayer.cs & $\begin{array}{l}\text { Audio player, also the background music player. All of the voice function to expand on this } \\
\text { basis. }\end{array}$ \\
\hline 8 & ConfigReader.cs & $\begin{array}{c}\text { Configuration file reader for reading configuration files, supply StepUnit. Cs generate } \\
\text { different data structure, ready for use. }\end{array}$ \\
\hline 9 & DelegateDefine.cs & $\begin{array}{c}\text { The callback base class, defines the format of the variety of the callback, convenient to } \\
\text { return different news and deal with different events. }\end{array}$ \\
\hline
\end{tabular}

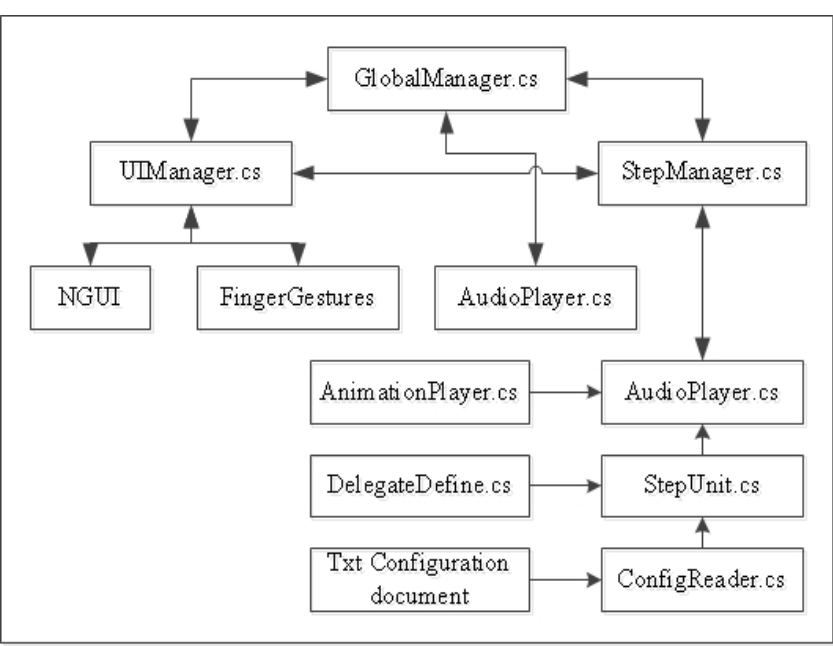

Figure 1 Class relationship diagram.

Various key class relationship of application is shown in Fig.1. GlobalManager.Cs, UIManager.Cs and StepManager.Cs is mutual coupling between the three. UIManager.Cs is mainly responsible for the response and handling UI message, at the same time also with StepManager.Cs is responsible for handling system messages together, and the results inform GlobalManager.Cs.

System deploies for each training mode the TXT file, including virtual resource object location, the virtual object animation step and every step of the animation start frame, end frame, and a series of information. Trainees once selected a pattern, the pattern of state will be recorded in global enumeration of GlobalManager.cs, and inform the UI Manager.Cs and StepManager.Cs operation mode of the current system. ConfigReader.Cs is responsible for reading the corresponding mode of TXT configuration files, and sends the data to StepUnit.Cs, generate a fixed data structure (firstFram lastFram, delayTime, description), stores in a linked list, ready for StepPlayer. Cs to use. If trained personnel operate right or need the animation, StepManager.Cs tell StepPlayer.Cs the desired animation. StepPlayer.cs use AnimationPlayer.cs playback unit corresponding to the list of animation to achieve interactive response.

Based on the structure, if need to join a new training course, only need to put the good animation, configured response TXT file, it can be added at any time.

\section{B. The Realization of Multitasking Gestures Interactions}

Another key technology of this system is multitasking gestures interactions. Gesture is mainly used to realize virtual environment navigation, touch UI virtual objects and operating equipment. So we need to determine the various tasks gestures according to the functional requirements.

Virtual environment navigation is to check the function of the virtual object through gestures. That means according to the need to change the camera's Angle of view, orientation and location. Several navigation gestures designed accordingly: single point to slide up and down or so, double refers to the relatively close to/relatively far from them. Single sliding realize rotation around the virtual object, double refers to slide up and down or so mobile virtual objects. Click on the button icon in the UI to operate UI, and use one hand click gesture.

In accordance with the need of gestures task, Unity3D specifically provides developers with two input object: touch and input, conveniently access to state of trainees to touch, including the touch position, away from the touch location. Using touch and input, through the combination between each variable can solve any single gesture events, but this approach is not practical, and not conducive to solve complex problems. Gestures plug-in FingerGestures3.0 is especially written for Unity. The plug-in layer makes gestures in the form of C\# proxy, including five default, and could easily listen to all kinds of gestures in the Unity event: the up and down or so four directions of sliding, press events, lift, mobile events, etc.

\section{THE REALIZATION OF THE SYSTEM}

On the basis of the above, the final the system also lies in the scene modeling and scene drive. System development process is shown in Fig.2.

To a large extent, the true extent of virtual object, system frame effect determines the immersion of the training. But should not be one-sided pursuit of the effect of the picture, 
without considering the degree of fluent software running on the platform. Therefore, when we make model using the 3ds Max 2012, for virtual object component interactions, a detailed modeling, not conversely. Textures virtual object completely get from the equipment acquisition, allowing trainees feel very real.

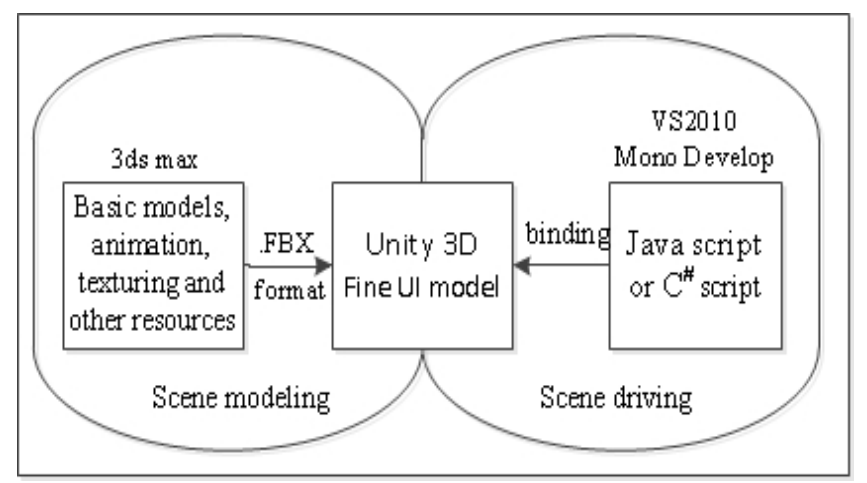

Figure 2 System development process.

After the virtual object is created, use .FBX plug-in from 3ds max2012 to export the virtual object, animation, texture resources .FBX file formats import Unity3D performed scene configuration, selection of flat-panel system version supports shader. System interface design using NGUI plugin, you can easily make simple and friendly interactive interface.

Scenario-driven mainly refers to the writing of script language, namely function code to write. In this system development, the main development language is C\#, you also need some useful scripting plug-ins, mainly NGUI, FingerGestures3.0 plug-in. Use Visual Studio2010 and Unity with Mono Develop for C\# or Java script script programming. The script is bound to the operation target or UI to achieve the scene drive, which can be edited to publish Web, PC or mobile terminal by Unity deployment ${ }^{[8]}$.

\section{CONCLUSION}

We adopt the design concept of 3d games to develop a portable virtual training system, with the help of the cross-platform development engine Unity $3 \mathrm{~d}$. Adopting the idea of three layer structure, through the C\# programming, establishes the general architecture of the system. Multitasking gestures are determined according to the design features and equipment operating requirements of the system. Study the method to realize the multitasking gestures. Finally realize the virtual training equipment by using the general system on a portable android platform, which meet the requirement of training. Compared with expensive immersive and enhanced virtual training systems, hardware requirements of the system is low, and the training is interesting, reducing development costs, which provides some reference for virtual operation on the portable platform with gesture touch technology.

\section{ACKNOWLEDGEMENT}

This research was financially supported by Wuhan N.C.O. Academy of P.L.A.

\section{REFERENCES}

[1] LI Wan,WANG Xuejun,CUI Xiaopeng,et al.Application of GL Studio and Vega Prime virtual training simulation system of a naval gun[J].Journal of Gun Launch Control,2010(2): 28-30.(in Chinese)

[2] PENG Liang,HUANG Xinhan. Missile combat virtual simulation system based on HLA and Vega Prime[J]. Journal of Central South University: Science and Technology, 2011, 42(4): 1015-1020. (in Chinese)

[3] WANG Xiancheng,LI Bo,LI Li.Research on key technology of virtual mintenance certain boat diesel engine based on Virtools 4.0[J].Journal of Academy of Armored Force Engineering, 2010, 24(2):27-3l(in Chinese)

[4] YANG Qingwen,FANG Shidong,YANG Guang,e t a1.Research on training simulation of techni que check for rocket launcher based on Virtools[J]. Computer Measurement\&Control, 2012,20(2): 407-410.(in Chinese)

[5] REN Guodong, CHEN Linhua,TAO Xuefeng,et a1.Virtual museum in formation visualization system basedon Unity $3 \mathrm{D}[\mathrm{J}]$.Computer Systems\&Applications,2013,29(9):87-90.(in Chinese)

[6] WU Bin, HUANG Zanzhen, GUO Xuefeng. Unity 4.x from the introduction to the master[M]. Beijing:China Railway Press,2013:1-13.(in Chinese)

[7] JIN Xizeng. Moble game development of Unity 3D[M]. Beijing:Tsinghua University Press, 2013:329-333.(in Chinese)

[8] WU Yafeng,YU Fuxing.Technology explanation and typical cases of game development by Unity 3D[M].Beijing:Posts and Telecom Press,2012:158-159.(in Chinese) 